\title{
Infinitely-Many Absorbing-State Nonequilibrium Phase Transitions
}

\author{
Frédéric van Wijland ${ }^{1,2}$ \\ ${ }^{1}$ Institute for Theoretical Physics, Postbus 80006, 3508 TA Utrecht, The Netherlands \\ ${ }^{2}$ Laboratoire de physique théorique, bâtiment 210, Université de Paris-Sud, 91405 Orsay cedex, France.
}

Received on 23 April, 2003

\begin{abstract}
We present a general field-theoretic strategy to analyze three connected families of continuous phase transitions which occur in nonequilibrium steady-states. We focus on transitions taking place between an active state and one absorbing state, when there exist an infinite number of such absorbing states. In such transitions the order parameter is coupled to an auxiliary field. Three situations arise according to whether the auxiliary field is diffusive and conserved, static and conserved, or finally static and not conserved.
\end{abstract}

\section{The ubiquity of absorbing-state transitions}

This overview is devoted to a study of nonequilibrium phase transitions taking place between the active and the absorbing state of a system, as some control parameter is varied across a threshold value. Such transitions are encountered in a variety of fields ranging from chemical kinetics to the spreading of computer viruses [1]. From a theoretical standpoint absorbing state transitions form natural counterparts to equilibrium phase transitions. The transition rates used in the stochastic dynamics employed to model the physical phenomenon under consideration do not satisfy detailed balance (with respect to an a priori defined energy function). In spite of this apparent freedom, the number of universality classes that the transition can fall into is incredibly small. Among known universality classes, that of directed percolation (DP) is by far the broadest. And indeed, in the absence of additional symmetries or conservation laws, as was conjectured twenty years ago by Grassberger [2], an absorbing state transition will invariably fall into the DP universality class. The interest in absorbing state transitions was further enhanced as Dickman and coworkers [3] established a one-to-one correspondence with self-organized critical systems (see [4] for a review on self-organized criticality). They were able to show that the scaling behavior observed there was entirely governed by an underlying nonequilibrium phase transition (which, as a side effect, somewhat tempers the mystics of SOC).

The study of exactly which microscopic ingredients make an absorbing state transition not belong to the DP class has almost grown into a field of its own. It was early realized that if the microscopic dynamics possesses additional conservation laws the universality class of the transition could be different. Discrete conservation laws, such as the conservation of the parity of the number of particles [5], are known to be driving the transition to an independent universality class (the Parity Conserving or Voter class [6]). A recent study attempts to provide a comprehensive table of all pos- sible transitions involving the dynamics of a single field [7]. Besides, the effect of a continuous symmetry was shown either to change the universality class of the transition $[8,9]$ or to simply destroy its continuous nature [10]. The continuous symmetry present in the systems studied there arose from a local conservation law.

An independent direction of research has focused on absorbing-state transitions in which the order parameter freezes into one among an infinity of absorbing states, but without any additional conservation law. The paradigmatic example of a system showing such a behavior is the pair contact process, initially introduced by Jensen and Dickman [11], for which Muñoz and coworkers [12] devised a convincing phenomenological picture that we shall later rely on for our analytic manipulations. The sensitivity of the DP class with respect to the coupling to an auxiliary field might actually provide a way out for explaining the difficulty of experimentally observing of the DP class [13], in spite of recent efforts (see [14] and references therein). Among the few effects that may lead a transition to depart from the directed percolation class we list quenched disorder and the coupling to an auxiliary field. The present works focuses on the latter (which was first formalized in those terms by Grassberger, Chaté and Rousseau [15]). We should also emphasize that the following exposition is a one-sided approach to those phenomena, relying solely on the fieldtheoretic approach, thus completely omitting the huge numerical efforts invested in the field.

The existence of an infinite number of absorbing states (in the large-system limit) and the coupling to an auxiliary field are the common characteristics to the microscopic models that we wish to investigate here. We will provide a full renormalization group picture of the phase transition at work in systems possessing an infinite number of absorbing states, with or without an additional conservation law. We shall rely on a combination of exact mappings and phenomenological Langevin equations approach as a starting point for the calculations. We begin by introducing the three families of models the critical behavior of which we wish to understand. Then we sketch the field-theoretic stragegy 
that we will follow on the simplest example of an epidemic. As we turn our attention to more complex models we shall identify which are the new key ingredients that pose technical difficulties. By doing so we come across an unexpected connection between the last two families of models we want to consider. The conclusion section lists some open problems with the present approach and gives directions for possible future works.

\section{Three families of processes}

\section{A. Spreading of an epidemic (SIS)}

A model for the spreading of an epidemic which is well-knwon to epidemiolgists is the so-called SusceptibleInfected-Susceptible model (SIS). The population is divided into two groups, the susceptible $A$ 's and the infected $B$ 's, whose interactions are contamination of an $A$ by a $B$ upon encounter,

$$
A+B \rightarrow B+B
$$

and spontaneous recovery of an infected individual,

$$
B \rightarrow A
$$

The motion of the individuals will be assumed to be diffusive, with diffusion constants $D_{A}$ and $D_{B}$ for the susceptible and the infected individuals, respectively. While the assumption of diffusive motion could itself be discussed (the very same reaction processes with underlying motion provided by a chaotic flow are used to model plancton population dynamics in the ocean [16]) we will take it for granted. The hope is that this simple assumption will apply to the majority of systems with such competitive rules.

When the average density of individuals $\rho$, which is obviously conserved by the above rules, is high enough, the infection survives indefinitely. At low density, on the contrary, the epidemic becomes extinct exponentially fast. And there exists a critical density threshold $\rho_{c}$ separating the two steady-states, i.e. the active one, with ongoing spreading, from the absorbing one in which the extinct epidemic cannot be revived. In the absorbing states, $A$ particles are freely diffusing.

The critical properties of, say the density of infected individuals, in the vicinity or at the density threshold were studied both analytically [8-10] and numerically [17-19], with a rich variety of behaviors. There is even still some controversy over the order of the transition in low space dimensions (mean-field predicts a continuous transition).

It is not hard to coin a mean-field phenomenological evolution equation for the order parameter field $\psi$,

$$
\partial_{t} \psi=D_{B} \Delta \psi+\left(\rho / \rho_{c}-1\right) \psi-g_{1} \psi^{2}
$$

where $g_{1}$ is a coarse-grained contamination rate. From this equation we deduce that the density of infected individuals $\psi$ in the steady-state, undergoes a continuous transition from an active state in which, as $\rho \rightarrow \rho_{c}^{+}, \psi \sim\left(\rho-\rho_{c}\right)^{\beta}$, to an absorbing state as $\rho \leq \rho_{c}$, with $\psi=0$. At the mean-field level $\beta=1$. Similarly the relaxation rate to the steady-state occurs over a typical time scale $\tau \sim \xi^{z}$ with $z=2$ and there is typical correlation length $\xi \sim\left|\rho-\rho_{c}\right|^{-\nu}$, with $\nu=1 / 2$. At the critical point the density decays as $\psi(t) \sim t^{-\delta}$ with $\delta=1$. This set of mean-field exponents will accurately describe the scaling properties of the transition whenever the space dimension is high enough to allow diffusion to quickly homogeneize space fluctuations. However, in low space dimensions, where random walks are recurrent or weakly transient, correlations between long-lasting fluctuations will play a role and the scaling behavior will be modified. In a region of linear size $\ell$ density fluctuations are of order $\ell^{d / 2}$, and thus relative density fluctuations are of the order $\ell^{-d / 2}$. From (3) we see that the reaction relaxation time goes roughly inversely proportional to the density fluctuations, hence the time it takes for the reaction to equilibrate over the region of size $\ell$ is of the order $\ell^{d / 2}$. But this meanfield reasoning holds only if diffusion has acted fast enough so as to erase space fluctuations over the domain of size $\ell$, which takes a time of the order $\ell^{2}$, and for mean-field to hold we must have $\ell^{2} \ll \ell^{d / 2}$, that is $d>4$.

The features which make of the SIS a system whose behavior is not of DP type are the following: the order parameter field is coupled to an auxiliary conserved field $\phi$ standing for the local density fluctuation of particles (independently of their $A$ or $B$ type). There exist an infinite number of absorbing states, but admittedly all of them are equivalent. In [20] Grassberger makes a distinction between fully ergodic absorbing states (such as the ones resulting from the epidemic becoming extinct) and those which are frozen in. We now turn to an example of the latter.

\section{B. Fixed Energy Sandpiles (FES)}

The second family of systems that we would like our analysis to encompass has, at first sight, no relationship with the above. Stochastic fixed energy sandpiles (FES), known as the Manna model [21, 3, 22], are defined as follows. Grains of sand are distributed on the sites of a lattice. Whenever a site is occupied by more than two grains, these are randomly distributed to the nearest neighbors. After a while, at sufficiently low grain density, all the sites will have less than two grains and the activity remains frozen in forever. At high grain density the toppling processes will keep occurring throughout the system, thus leaving a finite density of active -or toppling- sites in the infinite time limit. Of course, the system is supposed to be closed (the grains cannot escape though the boundaries). Identify now an active site with an infected individual $B$ and a still site with a susceptible $A$. Only the $B$ 's are now diffusing, but otherwise the reaction rules $(1,2)$ continue to roughly describe what is taking place in the system. Interestingly, and this is the important contribution of Vespignani and coworkers, a very large number of cellular automata can effectively be described by the SIS model in which the susceptibles are static. While this slight variation on the model present in the previous paragraph sounds innocuous, it will prove a dramatic change for te scaling properties. To get a first feeling of why it is so, we note that, as previously, the order parameter is coupled to a conserved field. But now this field is static, or rather its dynamics is slaved to that of the order parameter. We may expect, thus, that the fluctuations of this field will 
introduce memory effects, and lead to increased deviations to mean-field behavior. Again there exist an infinite number of absorbing states, but we do not know a priori what their distribution is. Pastor-Satorras and Vespignani [23] performed the first study of the epidemic with static healthy individuals, after the general characteristics of systems described by those schematic reaction rules had been identified by Rossi et al. [24] by studying two distinct automata, the Activated Random Walkers and the Conserved Transfer Threshold Process.

\section{Pair Contact Process (PCP)}

The third and final family of models we will bend over is embodied by the Pair Contact Process (PCP), a reaction involving a single species of static particles denoted by $C$. These excluding particles may either annihilate when on neighboring sites according to $C+C \rightarrow \emptyset$ or produce an offspring $C+C \rightarrow C+C+C$. But they do not diffuse. The order parameter of the transition is the density of pairs of nearest neighbor particles. At high branching rate the steady-state exhibits a finite density of particles, while at low branching the system eventually settles in a frozen state in which all particles are isolated. The order parameter of the transition is the local pair density. The connection between the PCP and the above two processes lies in the identification of a pair with a $B$ particle, and of an isolated particle with an $A$. Unfortunately there is no ready-to-use one-to-one mapping between a configuration of $A$ 's and $B$ 's and a configuration of the $C$ 's. One can draw a heuristic correspondence based on physical intuition, which can be written in a rather loose notation in a form reminiscent of the previous paragraphs:

$$
A+B \rightarrow B+B, \quad B \rightarrow \emptyset, \quad B \rightarrow A
$$

While there is obviously no particle conservation one may easily identify a branching process similar to the contamination one and two growth-limiting processes. Note also that the branching of a pair induces effective diffusion for the $B$ 's. A large number of systems, such as the dimer reaction [11], the dimer-dimer [25] and dimer-trimer [26] reactions, or the threshold transfer process [27] are modeled by the above mechanism.

\section{Field-theoretic techniques: a uni- fied treatment}

\section{A. Action for the epidemics}

There are well-established techniques [28] for mapping a reaction-diffusion process in which particles diffuse, branch, annihilate, and possibly exclude each other, onto a field-theory that exactly encodes the microscopic dynamics. We will not review how such a mapping is achieved. It suffices to know that the resulting field theory features, for each species of particles, a density field, the first moment of which gives the local density of particles, and a response field (usually bearing an overbar) with no straightforward physical interpretation. Denoting by $\psi$ the order parameter field and by $\phi$ the local density fluctuation we have the following action

$$
\begin{aligned}
S[\bar{\psi}, \psi, \bar{\phi}, \phi]=\int \mathrm{d}^{d} x \mathrm{~d} t[ & \bar{\psi}\left(\partial_{t}+\sigma-D_{B} \Delta\right) \psi \\
& +\bar{\phi}\left(\partial_{t}-D_{A} \Delta\right) \phi \\
& +\bar{\phi}\left(D_{A}-D_{B}\right) \Delta \psi \\
& \left.+\bar{\psi} \psi^{2}-\bar{\psi}^{2} \psi+\bar{\psi} \psi(\bar{\phi}+\phi)\right]
\end{aligned}
$$

where we have omitted higher order terms in the fields and have not specified any names for the interaction vertices. Note that the free field $\bar{\phi}, \phi$ could be integrated out to yield an effective action for the order parameter $\psi$ and its response field $\bar{\psi}$ alone. The renormalization group analysis of (5) was performed [8-10] with the following results : the coupling of the order parameter to an auxiliary diffusive and conserved field drives the transition to two universality classes different from the DP one according to whether $0<D_{A}<D_{B}$ or $D_{A}=D_{B}$. Even more surprisingly, the continuous phase transition is outweighed by a first order one below the upper critical dimension when $0<D_{B}<D_{A}$. While a first-order transition was indeed observed in a two-dimensional system, it has been both ruled out [18, 29] and confirmed [30] in one dimension. In the following table we recall the results for the critical exponents given to first order in $\varepsilon=4-d$ (a star superscript indicates a result holding to all orders in $\varepsilon$ ) and provide for comparison the directed percolation expression [31]. The exponents $\eta$ and $\bar{\eta}$ are the anomalous dimensions of the $\psi$ and $\bar{\psi}$ fields, respectively, defined as $\psi_{\mathrm{R}} \sim \ell^{-\frac{d+\eta}{2}}$ and $\bar{\psi}_{\mathrm{R}} \sim \ell^{-\frac{d+\bar{\eta}}{2}}$, where $\ell$ is a length scale.

\begin{tabular}{|c|c|c|c|}
\hline exponent & $D_{A}=D_{B}$ & $D_{A}<D_{B}$ & DP \\
\hline$z$ & $2^{*}$ & $2^{*}$ & $2-\frac{\varepsilon}{12}$ \\
\hline$\nu^{-1}$ & $2-\frac{\varepsilon}{2}{ }^{*}$ & $2-\frac{\varepsilon}{2}^{*}$ & $2-\frac{\varepsilon}{4}$ \\
\hline$\eta$ & $-\frac{\varepsilon}{8}$ & $0^{*}$ & $-\frac{\varepsilon}{6}$ \\
\hline $\bar{\eta}$ & $-\frac{\varepsilon}{8}$ & $-0.313 \varepsilon$ & $-\frac{\varepsilon}{6}$ \\
\hline$\beta=\nu \frac{d+\eta}{2}$ & $1-\frac{\varepsilon}{8}$ & $1^{*}$ & $1-\frac{\varepsilon}{6}$ \\
\hline
\end{tabular}

It is worth commenting on Table (6): several exponents appear to be given to all orders in $\varepsilon$. That the $z$ exponent takes the value 2 is understable since the conserved and freely diffusive field $\phi$ imposes its (slow) relaxation scale. Hence the superdiffusive behavior observed in DP $(z<2)$ when it is not coupled to any auxiliary field is overcome by the slowest relaxing modes, which happen to be freely diffusive modes (with diffusion constant $D_{A}$ ). This is a very robust property that can be seen to hold even if we took the omitted quartic terms in (5) into account. It is rooted in the absence of renormalization of the $\phi$ propagator. The exactness of the correlation length exponent $\nu=2 / d$ has a very different origin. A shift of the density field $\phi$ by a constant has the same effect as shifting the $\psi$ field mass term. This implies a exact identity between vertex function, which carries over to the renormalized quantities, thus yielding an additional relationship between exponents, namely that $\nu^{-1}$ equals the scaling 
dimension of $\phi$, which is $d / 2$. Unfortunately this continuous symmetry is broken by quartic terms whose effect in low-dimensions is ill-controled. This has led to some debate in the literature $[33,32,18]$. Quite understanbly, the exact knowledge of some exponents (if blindly taken for granted) eases numerical analysis and yields higher precision results on the remaining exponents.

\section{B. One-loop-expansion for FES}

One crucial difference between the SIS and the FES is that the $A$ particles become static, which can be achieved simply by setting $D_{A}=0$ in (5). Note that an immediate consequence of setting the auxiliary field diffusion constant to zero is that it ceases to impose its own dynamic scale (in particular, we should now expect that the order parameter field $\psi$ will indeed exhibit superdiffusive behavior, and that $\phi$ will follow). The total density field $\phi$ has its dynamics slaved to the local fluctuations in the order parameter $\psi$, as can be see by writing the equation of state for $\phi$ :

$$
\partial_{t}\langle\phi\rangle=D_{B} \Delta\langle\psi\rangle
$$

This means that the modes of $\phi$ describing the short-scale fluctuations verify $\phi(\mathbf{q}, t) \sim-\mathbf{q}^{2} \int^{t} \mathrm{~d} \tau \psi(\mathbf{q}, \tau)$. When integrating out the $\bar{\phi}, \phi$ fields from the action (5) one is left with effective interaction of the form

$$
g_{4} \int \mathrm{d}^{d} x \mathrm{~d} t \bar{\psi} \psi(\mathbf{x}, t) \int^{t} \mathrm{~d} t^{\prime} \Delta_{\mathbf{x}} \psi\left(\mathbf{x}, t^{\prime}\right)
$$

But at small distances (large q's) there is no difference between the $g_{4}$ vertex and

$$
g_{3} \int \mathrm{d}^{d} x \mathrm{~d} t \bar{\psi} \psi(\mathbf{x}, t) \int^{t} \mathrm{~d} t^{\prime} \psi\left(\mathbf{x}, t^{\prime}\right)
$$

Thus it is not surprising that a $g_{3}$-like vertex is generated already at one loop, as can be seen on the Feynman diagram depicted in Fig. 1 .

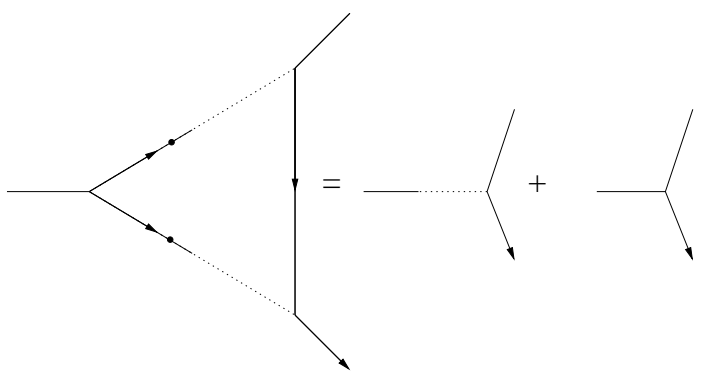

Figure 1 . The one-loop diagram combines the $g_{4}$ and $g_{2}$ vertices to yield effective $g_{3}$ and $g_{1}$ couplings. A black dot on a leg means that the vertex is proportional to the square of the momentum flowing through the leg. The dotted line means that the dangling leg is at time earlier than the other two making up the vertex.

The fact that such terms are generated would seem to be in conflict with the local conservation law. But there is no contradiction as other terms are generated, which will turn out to be irrelevant from the renormalization group point of view, yet they will take care of preserving this essential property. We now rewrite the effective action for the order parameter field only:

$$
\begin{aligned}
S[\bar{\psi}, \psi]=\int \mathrm{d}^{d} x \mathrm{~d} t\left[\bar{\psi}\left(\partial_{t}+\lambda \tau-\lambda \Delta\right) \psi\right. & g_{1} \bar{\psi} \psi^{2}-g_{2} \bar{\psi}^{2} \psi \\
& +g_{3} \bar{\psi} \psi \int \mathrm{d} t^{\prime} \psi\left(\mathbf{x}, t^{\prime}\right) \\
& -g_{4} \bar{\psi} \psi \int \mathrm{d} t^{\prime} \Delta \psi\left(\mathbf{x}, t^{\prime}\right) \\
& \left.-g_{5} \bar{\psi} \psi \int \mathrm{d} t^{\prime} \bar{\psi} \psi\left(\mathbf{x}, t^{\prime}\right)\right]
\end{aligned}
$$

The $g_{1}$ and $g_{2}$ vertices alone make up the directed percolation action. In fact, in all members of the FES family, vertices of the form $\int \mathrm{d}^{d} x \mathrm{~d} t \bar{\psi} \psi\left[\int^{t} \mathrm{~d} t^{\prime} \psi\left(t^{\prime}\right)\right]^{n}$ are generated at one loop. To illustrate this state of affairs, in Fig. 2 we draw the one-loop diagram generating the $n=2$ term.



Figure 2. Two $g_{3}$ vertices are combined with a $g_{5}$ vertex to yield an effective $\int \mathrm{d}^{d} x \mathrm{~d} t \bar{\psi} \psi\left[\int^{t} \mathrm{~d} t^{\prime} \psi\left(t^{\prime}\right)\right]^{2}$ interaction.

In the next section it is explained how to make physical sense of the action (10) and to extract physical quantities from it.

\section{The special PCP case}

In the PCP the microscopic dynamics is quite different. Following the suggestion of Muñoz [12], it is not hard to see that the directed percolation action has to be supplemented by an additional interaction term of the form

$$
\delta S_{\mathrm{PCP}}[\bar{\psi}, \psi]=-\int \mathrm{d}^{d} x \mathrm{~d} t \bar{\psi} \psi(\mathbf{x}, t)\left[\mathrm{e}^{-\lambda \int_{0}^{t} \mathrm{~d} t^{\prime} \psi\left(\mathbf{x}, t^{\prime}\right)}-1\right]
$$

Note that subtracting -1 in the bracket in the right-handside of (11) amounts to shifting the mass term of the field theory (which now vanishes at the mean-field critical point). The mass term being positive, this means that we are working in the absorbing phase. In other words, the order parameter relaxes exponentially fast to zero. We choose to perform our analysis with a positive mass, and we shall carry the necessary renormalizations in the absorbing phase, as is done in all previous renormalization group studies of absorbing state transitions. Working in the active phase would lead to the same renormalization factors, but would imply following a much more complicated path with exactly the same outcome.

One could believe that the present exponential term will not contribute to determining the anomalous scaling properties of the phase transition at work by arguing as follows. The order parameter will tend to a constant value and hence 
this term will be suppressed exponentially fast. But this reasoning involves fixing the mass and letting time to infinity. We are interested in the reverse limit in which time is much shorter than any typical critical time so that the critical fluctuations can develop (but of course much larger than microscopic time scales). And those limits do not commute. All the terms making up the series expansion of $\delta S_{\mathrm{PCP}}$ are relevant at the Gaussian fixed point (the $n$ 's power of $\lambda$ has bare dimension $2+n \varepsilon$, which indicates that those terms will constitute relevant perturbation of the directed percolation $\mathcal{O}(\varepsilon)$ fixed point.

The nature of short-time and short-distance singularities (UV divergencies) dictates the universality class that a phase transition falls into. This is quite counter-intuitive since a phase transition is a large-scale collective phenomenon. And indeed renormalization is not but a refined coarse-graining procedure which integrates out the short-time and shortdistance degrees of freedom, and which will carry these pieces of information over to the macroscopic degrees of freedom (the global order parameter, for instance).

\section{Analysis}

In order to study UV divergencies we follow the strategy outlines in [34] and we expand $\delta S_{\mathrm{PCP}}$ in powers of $\lambda$. Then a quick inspection at the one-loop graphs tells us that the new upper critical dimension is $d_{c}=6$, below which the theory is super-renormalizable, and above which mean-field applies. From here on the notation $\varepsilon$ stands for the deviation of the space dimension with respect to the new upper critical dimension $d_{c}=6: \varepsilon=6-d$. It is instructive to write $\delta S_{\mathrm{PCP}}$ in the form

$$
\begin{aligned}
\delta S_{\mathrm{PCP}}[\bar{\psi}, \psi]= & \lambda \int \mathrm{d}^{d} x \mathrm{~d} t \bar{\psi} \psi(\mathbf{x}, t) \int_{0}^{t} \mathrm{~d} t^{\prime} \psi\left(\mathbf{x}, t^{\prime}\right) \\
& -\int \mathrm{d}^{d} x \mathrm{~d} t \bar{\psi} \psi(\mathbf{x}, t) \times \\
& {\left[\mathrm{e}^{-\lambda \int_{0}^{t} \mathrm{~d} t^{\prime} \psi\left(\mathbf{x}, t^{\prime}\right)}-1+\lambda \int_{0}^{t} \mathrm{~d} t^{\prime} \psi\left(\mathbf{x}, t^{\prime}\right)\right] }
\end{aligned}
$$

because now the brackets in the right-hand-side contain terms which are irrelevant in the renormalization group sense. A word of caution is needed here: the unfortunate wording irrelevant does not mean those terms are irrelevant physics-wise, and indeed by throwing them away we would simply lose the physical mechanism at work in the PCP (there would simply be no more phase transition). This merely expresses that those additional terms do not introduce corrections to the large scale effective couplings. There are many instances of systems described by a field theory in which RG-wise irrelevant terms govern the phase diagram, but not the anomalous scaling properties, both in equilibrium (see the review by Amit and Peliti [35]) and out of equilibrium (see Janssen and Schmittmann [36] for the example of a driven diffusive system or Deloubrière al. [37], more recently, for providing the analysis of the Pair Contact Process with Diffusion). All powers of $\lambda$ higher than two therefore constitute dangerously irrelevant terms.

However, having in mind that now the upper critical dimension is shifted up to $d_{c}=6$, we see that also $g_{1}$ is a dangerously irrelevant coupling to the extent that, as shown by Muñoz and coworkers [12], it eventually controls the dynamics of the order parameter by playing the role of the leading nonlinear growth-limiting coupling (at late times).

There are now two separate problems. The first one is to renormalize the field-theory with the $g_{5} \bar{\psi} \psi \int^{t} \mathrm{~d} t^{\prime} \psi\left(t^{\prime}\right)$ vertex. But this has already being done almost twenty years ago by Janssen [38] and Grassberger and Cardy [39] (this is the same field theory that describes dynamical percolation). And then one must compute the scaling dimension of the dangerously irrelevant operators at the dynamical percolation fixed point. The second issue is how to extract the scaling behavior of physical observables knowing that the usual scaling assumption breaks down. Again it may look surprising that the universality class is that of dynamical percolation, a process with no absorbing state transition. This is because in the PCP the phase transition is driven by irrelevant couplings (which are absent in dynamical percolation).

The scaling dimension of $g_{1}$ at the dynamical percolation fixed point is found to be $y_{g_{1}}=-2-\frac{\varepsilon}{7}$. At scale $b \gg 1$, the effective $g_{1}(b)$ behaves as $b^{y_{g_{1}}}$. And then we use the mean-field scaling function's dependence in $g_{1}$ (which depends on $g_{1}$ as $\left.1 / g_{1}\right)$ which is correct to first order in $\varepsilon=6-d$. The conclusion is that

$$
\langle\psi\rangle \sim b^{-\frac{d+2+\eta}{2}} \mathcal{F}\left(b^{1 / \nu}|\sigma|, b^{-z} t, g_{1}(b)\right)
$$

with $\mathcal{F}(x, y, z) \stackrel{z \rightarrow 0}{\propto} \frac{F(x, y)}{z}$ to leading order in $\varepsilon$. We can therefore extract the following critical exponents

$$
\beta=1-\frac{3}{14} \varepsilon, \quad \delta=1-\frac{1}{4} \varepsilon
$$

which are valid to first order in $\varepsilon$. We have used the dynamical percolation expressions for the correlation time and correlation length exponents, $z=2-\frac{\varepsilon}{6}$ and $\nu^{-1}=2-\frac{5}{21} \varepsilon$.

\section{Conclusions}

One of the major drawbacks of the analysis presented here, as far as the FES and PCP-like systems are concerned, is the absence of agreement with numerical simulations. That there is disagreement with the dynamical percolation picture in one-dimensional systems is not too surprising owing to the triviality of percolation in $d=1$. This signals that there is very likely an intermediate dimension below which the universality class that we have described shifts to some other class. It could even be that FES and PCP systems behave differently in low space dimensions. As usual with expansions in the vicinity of the upper critical dimension, few results lend themselves to direct comparison with the simulations. We shall not try to review the numerical status of the systems considered throughout this paper, but we mention that the general belief is that the upper critical dimension, both for the FES and PCP-like systems is $d_{c}=4$, and not $d_{c}=6$ as advocated here. We refer the interested reader to [40].

We would like to mention a list of interesting problems. In all three families of processes that we have considered 
there is competition between a one-particle branching process and nonlinear growth limiting processes. It was recently argued that binary spreading processes (first introduced by [41]), that is reactions in which branching only occurs by pairs, would lead absorbing state transitions to a universality class different from that of directed percolation. While there is not even any consensus on that, we speculate that it is indeed so [37], at least in the case of the Pair Contact Process with Diffusion (PCPD). In the PCPD there are two absorbing states (degenerate in the large size limit), but coupling it to an auxiliary field, possibly conserved and/or static, might reveal a rich variety of behaviors [42]. We leave this and other issues for future work.

\section{Ackowledgment}

The research presented here arose from direct collaborations and evergoing, and sometimes disagreeing, discussions with H.J. Hilhorst, K. Oerding, A. Vespignani, H. Chaté and M.A. Muñoz, who are all gratefully acknowledged. The author thanks the Fundamenteel Onderzoek der Materie (FOM) and the Lorentz Fonds for financial support.

\section{References}

[1] B. Chopard and M. Droz, Cellular automaton modeling of physical systems (Cambridge University Press, Cambridge, 1998); J. Marro and R. Dickman, Nonequilibrium phase transitions in lattice models (Cambridge University Press, Cambridge, 1999); H. Hinrichsen, Nonequilibrium Critical Phenomena and Phase Transitions into Absorbing States, Advances in Physics 49, 815 (2000); G. Ódor, Phase transition universality classes of classical, nonequilibrium systems (cond-mat/0205644).

[2] P. Grassberger and A. de la Torre, Ann. Phys. (N.Y.) 122, 373 (1979).

[3] R. Dickman, A. Vespignani, and S. Zapperi, Phys. Rev. E 57, 5095 (1998); A. Vespignani, R. Dickman, M. A. Muñoz, and S. Zapperi, Phys. Rev. Lett. 81, 5676 (1998).

[4] H. J. Jensen, Self-Organized Criticality (Cambridge University Press, Cambridge, 1998).

[5] J.L. Cardy and U.C. Täuber, Phys. Rev. Lett. 77, 4780 (1996) and J. Stat. Phys. 90, 1 (1998).

[6] I. Dornic, H. Chaté, J. Chave and H. Hinrichsen, Phys. Rev. Lett. 87, 045701 (2001)

[7] H. Chaté and J. Kockelkoren, Phys. Rev. Lett. 90, 125701 (2003).

[8] R. Kree, B. Schaub, and B. Schmittmann, Phys. Rev. A 39, 2214 (1989).

[9] F. van Wijland, K. Oerding, and H.J. Hilhorst, Physica A 251, 179 (1998).

[10] K. Oerding, F. van Wijland, J.-P. Leroy and H.J. Hilhorst, J. Stat. Phys. 99, 1365 (2000).

[11] I. Jensen and R. Dickman, Phys. Rev. E 48, 1710 (1993).

[12] M.A. Muñoz, G. Grinstein, R. Dickman and R. Livi, Phys. Rev. Lett. 76, 451 (1996) and Physica D 103, 485 (1997); M.A. Muñoz, G. Grinstein and R. Dickman, J. Stat. Phys. 91, 541 (1998).
[13] H. Hinrichsen, Braz. J. Phys. 30, 69 (2000).

[14] P. Rupp, R. Richter and I. Rehberg, Phys. Re. E 67, 036209 (2003).

[15] P. Grassberger, H. Chaté and G. Rousseau, Phys. Rev. E 55, 2488 (1997).

[16] G. Károlyi, Á. Péntek, Z. Toroczkai, T. Tél, and C. Grebogi, Phys. Rev E 59, 5468 (1999).

[17] J.E. de Freitas, L.S. Lucena, L.R. da Silva, and H.J. Hilhorst, Phys. Rev. E 61, 6330 (2000).

[18] U.L. Fülco, D.N. Messias, and M.L. Lyra, Physica A 295, 49 (2001) and Phys. Rev. E 63, 066118 (2001).

[19] E. Perlsman and S. Havlin, Europhys. Lett. 58, 176 (2002).

[20] P. Grassberger, J. Stat. Phys. 79, 13 (1995).

[21] S. S. Manna, J. Phys. A 24, L363 (1991); D. Dhar, Physica A 263, 4 (1999).

[22] A. Vespignani, R. Dickman, M. A. Muñoz, and S. Zapperi, Phys. Rev. E 62, 4564 (2000); cond-mat/0003285.

[23] R. Pastor-Satorras and A. Vespignani, Phys. Rev. E 62, R5875 (2000) and Eur. Phys. J. B 19, 583 (2001).

[24] M. Rossi, R. Pastor-Satorras, and A. Vespignani, Phys. Rev. Lett. 85, 1803 (2000).

[25] E.V. Albano, J. Phys. A 25, 2557 (1992); I. Jensen, Phys. Rev. Lett. 70, 1465 (1993).

[26] J. Kohler and D. ben-Avraham, J. Phys. A 24, L621 (1991).

[27] J.F.F. Mendes, R. Dickman, M. Henkel, and M. C. Marques, J. Phys. A 273019 (1994).

[28] M. Doi, J. Phys. A9, 1465 (1976).

[29] H. Hinrichsen, e-print cond-mat/0006212, First-order transitions in fluctuating 1+1-dimensional nonequilibrium systems, unpublished.

[30] A. Barrat and A. Vespignani, unpublished.

[31] H.K. Janssen, Z. Phys. B 42, 151 (1981).

[32] J.E. de Freitas, L.S. Lucena, L.R. da Silva, and H.J. Hilhorst, Phys. Rev. E 64, 058102 (2001).

[33] H.K. Janssen, Phys. Rev. E 64, 058101 (2001).

[34] F. van Wijland, Phys. Rev. Lett. 89, 190602 (2002).

[35] D.J. Amit and L. Peliti, Ann. Phys. (NY) 140207 (1982).

[36] H.K. Janssen and B. Schmittmann, Z. Phys. B 64503 (1986).

[37] O. Deloubrière, U.C. Täuber and F. van Wijland, to be submitted.

[38] H.K. Janssen, Z. Phys. B 58311 (1985).

[39] J.L. Cardy, J. Phys. A 16, L709 (1983) and P. Grassberger and J.L. Cardy, J. Phys. A 18, 267 (1985).

[40] S. Lübeck, Phys. Rev. E 64, 016123 (2001); R. Dickman, M. Alava, M.A. Muñoz, J. Peltola, A. Vespigani and S. Zapperi, Phys. Rev. E 64, 056104 (2001); R. Dickman, T. Tomé and M.J. de Oliveira, Phys. Rev. E 66016111 (2002).

[41] P. Grassberger, Z. Phys. B 47, 365 (1982).

[42] H. Chaté and J. Kockelkoren, Absorbing phase transitions with coupling to a static field and a conservation law, preprint cond-mat/0306039. 